\title{
Reconstruction of a kinetic k-essence Lagrangian from a modified of dark energy equation of state
}

\author{
Víctor H. Cárdenas ${ }^{1, \mathrm{a}}$, Norman Cruz ${ }^{2, \mathrm{~b}}$, Sebastian Muñoz ${ }^{3, \mathrm{c}}$, J. R. Villanueva ${ }^{1, \mathrm{~d}}$ \\ ${ }^{1}$ Instituto de Física y Astronomía, Universidad de Valparaíso, Avenida Gran Bretaña 1111, Casilla 5030, Valparaiso, Chile \\ 2 Departamento de Física, Universidad de Santiago de Chile, Avenida Ecuador 3493, Casilla 307, Santiago, Chile \\ ${ }^{3}$ Departamento de Matemática y Ciencia de la Computación, Universidad de Santiago de Chile, Las Sophoras 173, Santiago, Chile
}

Received: 10 April 2018 / Accepted: 13 July 2018 / Published online: 21 July 2018

(C) The Author(s) 2018

\begin{abstract}
In this paper the Lagrangian density of a purely kinetic k-essence model that describes the behavior of dark energy described by four parameterized equations of state proposed by Cooray and Huterer (Astrophys J 513:L95, 1999), Zhang and Wu (Mod Phys Lett A 27:1250030, 2012), Linder (Phys Rev Lett 90:091301, 2003), Efstathiou (Mon Not R Astron Soc 310:842, 2000), and Feng and Lu (J Cosmol Astropart Phys 1111:34, 2011) has been reconstructed. This reconstruction is performed using the method outlined by de Putter and Linder (Astropart Phys 28:263, 2007), which makes it possible to solve the equations that relate the Lagrangian density of the k-essence with the given equation of state (EoS) numerically. Finally, we discuss the observational constraints for the models based on 1049 SNIa data points from the Pantheon data set compiled by Scolnic et al. (Astrophys J 859(2):101, 2018)
\end{abstract}

\section{Contents}

1 Introduction . . . . . . . . . . . . . . 1

2 Kinetic k-essence . . . . . . . . . . . . . 2

3 New parametrizations and kinetic k-essence Lagrangians .............................. 3

4 Observational constraints . . . . . . . . . 4

4.1 Model A . . . . . . . . . . . . . . . . . . . 5

4.2 Model B . . . . . . . . . . . . . . . . . . . 6

4.3 Model C . . . . . . . . . . . . . . . . . . . 6

4.4 Model D . . . . . . . . . . . . . . . . . 7

5 Conclusions and final remarks . . . . . . . . 7

References................. . . 8

a e-mail: victor.cardenas@uv.cl

b e-mail: norman.cruz@usach.cl

c e-mail: sebastian.munozc@usach.cl

d e-mail: jose.villanueva@uv.cl

\section{Introduction}

It is well known that the analysis of the luminosity-redshift relation for distant Type-SnIa supernovae suggested that the universe undergoes an accelerated expansion. This discovery motivated scientists to propose models that would attribute these behaviors in the universe responsible for this expansion to an unknown energy component called dark energy, which is homogeneously distributed in the universe, having negative pressure. To explain the observed accelerated expansion of the universe, the simplest solution was Einstein's idea of a vacuum energy, namely the cosmological constant $\Lambda$. A small positive cosmological constant has been supported by a number of observations. Indeed, the cosmological constant is a perfect fit to the dark energy data. An important question related to the cosmological constant is the fact that the energy densities of dark energy and dark matter are now comparable. This is called the coincidence problem. From the cosmological constant problem we are motivated to find an alternative explanation for dark energy. By modifying the left-hand side of Einstein's equation, we get the modified gravity models. The idea of modified matter models is that the energy-momentum tensor $T_{\mu \nu}$ contains exotic matter, which provides a negative pressure, which consists of a canonical scalar field with a standard Lagrangian of the form $L=X-V(\phi)$. Modifying this canonical kinetic energy term to non-linear kinetic terms, the non-linear kinetic energy of the scalar field can drive the negative pressure without the help of a field potential. These models are called kinetic kessence models; they consist of a scalar field described by a Lagrangian of the form $L=F(X, \phi)$ (see [1-5]). This generalization of the canonical scalar field models can give rise to new dynamics not possible in quintessence. The nonlinear kinetic energy terms are thought to be small and usually are ignored because the Hubble expansion dampens the kinetic energy density over time. But what happens if there 
is a dynamical attractor solution which forces the non-linear terms to remain non-negligible? This is the main idea of the $\mathrm{k}$-essence models. In the context of cosmology, k-essence was first studied as a model for inflation (k-inflation) [6], and due to its dynamics, increasing interest has been devoted to it in cosmological investigations. In this paper, we restrict ourselves to a particular case of kinetic k-essence, which is to take only kinetic terms, i.e., assume that the Lagrangian is of the form $L=F(X)$ [7]. Such scalar fields can be interpreted as barotropic perfect fluids. The classification of scalar field models that satisfy the condition of a barotropic perfect fluid are detailed in [9]. In [10] the physical stability restrictions for $C_{\mathrm{s}}^{2}$ are discussed, as well as the dynamics of four types of dark energy (EoS) models for the behavior of $\mathrm{k}$ essence and quintessence. In [11] the behavior of a model is addressed where the equation of state is a power law of the kinetic energy, $X\left(\omega=\omega_{0} X^{\alpha}\right)$, as are the conditions needed to have accelerated phases. Variable EoSs were considered in [12], where $\omega(z)$ was written as $\omega_{0}+z(\mathrm{~d} w / \mathrm{d} z)_{0}$, and by means of gravitational lensing, the expected accuracy to which today's equation of state $\omega_{0}$ and its rate of change $(\mathrm{d} w / \mathrm{d} z)_{0}$ can be simultaneously constrained was studied. In [14], from the joint analysis of four observations ( $\mathrm{SNe}+\mathrm{BAO}$ $+\mathrm{CMB}+\mathrm{H} 0$ ), constraints for the time-varying dark energy $\operatorname{EoS} \omega(z)$ were obtained, where $\omega(z)$ was parameterized via two parameters and some of them depend on a given extra parameter. A classification into two types of models for the suitable $\omega(z)$ was made according to the boundary behavior and the local extreme point of the $\operatorname{EoS} \omega(z)$.

As we have mentioned, this paper is devoted to presenting a method to reconstruct the Lagrangian of kinetic k-essence , using as initial information a specific parametrization of the EoS, the dark energy of the form $\omega=\omega(a)$. To do so, this paper is organized as follows: in Sect. 2 we review the main aspects of the kinetic k-essence model as a dark energy model. In Sect. 3 we develop the parameterizations that justify the model presented by Efstathiou, which modify the original one presented in [17]. Also, the evolution of these parameterizations, for the Lagrangian and for $X$ as a function of the scale factor, are found. Then, by defining the general relation $\frac{\mathrm{d} a}{\mathrm{~d} X}=\mathscr{H}(a, X)$, the kinetic k-essence Lagrangian is constructed, followed by an analysis of the behavior of the generating function $F$. In Sect. 4 we use the parameterizations defined above to obtain the best reconstruction of $F(X)$ based on observational data, consisting of 1049 SNIa data points from the Pantheon data set compiled in [28]. Finally, in Sect. 5 we end the work with final remarks and conclusions.

\section{Kinetic k-essence}

Our main goal is to investigate a dark energy model described by an effective minimally coupled scalar field $\phi$ with a non- canonical kinetic term, specifically to the so-called purely kinetic k-essence model, in which the Lagrangian density $\mathscr{L}=-F(X)$ depends only on the kinetic terms $X=$ $\frac{1}{2} \partial_{\mu} \phi \partial^{\mu} \phi$. This model can be obtained from the action for a k-essence field minimally coupled to gravity, given by

$S=-\int \mathrm{d}^{4} x \sqrt{-g}\left(-F(X)+\frac{1}{2} R\right)$,

in the background of an homogeneous and isotropic flat universe described by the Friedmann-Lemaître-RobertsonWalker metric:

$\mathrm{d} s^{2}=\mathrm{d} t^{2}-a(t)^{2}\left[\mathrm{~d} r^{2}+r^{2}\left(\mathrm{~d} \theta^{2}+\sin ^{2} \theta \mathrm{d} \phi^{2}\right)\right]$.

Unless otherwise stated, we consider $\phi$ to be smooth on the scales of interest so that $X=\frac{1}{2} \dot{\phi}^{2}$. The energy-momentum tensor derived from the action (1) allows us to identify the energy density $\rho$ and pressure $p$ with the expressions

$\rho=2 X F_{X}-F$

and

$p=F$,

where $F_{X} \equiv \mathrm{d} F / \mathrm{d} X$. Assuming that the energy density is a positive quantity, we demand that $2 X F_{X}-F \geq 0$. Therefore, with $F>0$, the parameter of the equation of state becomes

$\omega=\frac{p}{\rho}=\frac{F}{2 X F_{X}-F}$.

An important result relating $F, X$, and the scale factor $a$ is obtained when the scalar potential becomes constant, so the equations of motion yield the following expression [7]:

$X F_{X}^{2}=\kappa a^{-6}$,

where $\kappa$ is a constant of integration. This solution was previously derived, in a slightly different form, by Chimento in [8]. Thus, given any form of $F(X)$, the above equation gives the evolution of $X$ as a function of the scale factor $a$, and then, by substitution of this solution into Eqs. (3)-(4), we can obtain the evolution of all physical quantities of interest.

The square of the adiabatic speed of sound is obtained from the relation [23]

$c_{\mathrm{s}}^{2}=\frac{\mathrm{d} p / \mathrm{d} X}{\mathrm{~d} \rho / \mathrm{d} X}=\frac{F_{X}}{F_{X}+2 X F_{X X}}=\frac{\omega^{\prime}-3 \omega(1+\omega)}{3(1+\omega)}$,

where $F_{X X} \equiv \mathrm{d}^{2} F / \mathrm{d} X^{2}$, and a prime denotes differentiation with respect to $\ln a$. Notice that Eq. (7) imposes two conditions: the first one, since the speed of sound is a real quantity, 
we demand that $\omega^{\prime}-3 \omega(1+\omega) \geq 0$ [10]. The second one comes from the fact that the speed of sound is slower than the speed of light (which is equal to 1 in this work), so we must demand that $\omega^{\prime}-3 \omega(1+\omega) \leq 3(1+\omega)$. Finally, notice that, in general, the adiabatic speed of sound $c_{\mathrm{s}}$ does not coincide with the speed of propagation of scalar perturbations. However, in the kinetic k-essence scenario this statement does not work and these two speeds match, which implies that it is possible to describe a scalar field by a perfect fluid and vice versa [9].

Now, by combining Eqs. (4) and (5), it is possible to show that

$X^{2} F_{X}^{2}=\left(\frac{1+\omega}{2 \omega}\right)^{2} p^{2}$

and then using the following identities:

$$
\begin{aligned}
\frac{1+\omega}{\omega} & =\frac{p+\rho}{p}, \\
p^{\prime} & =\left[\omega^{\prime}-3 \omega(1+\omega)\right] \rho,
\end{aligned}
$$

we obtain the following equations for $X$ and $F$ in terms of the scale factor:

$X^{\prime}=-6 c_{\mathrm{s}}^{2} X$,

$F^{\prime}=3\left(\frac{1+\omega}{\omega}\right) c_{\mathrm{s}}^{2} F$.

Since the EoS parameter $\omega$ depends on the scale factor $a$, we introduce it here in the model to consider. Finally, by solving Eqs. (11) and (12), we can write

$F(X)=C \frac{\omega(a(X))}{a^{3}(X)(1+\omega(a(X)))} \sqrt{X}$.

Obviously, the main difficulty is to invert the expression for $X(a)$, so we will proceed by a numerical approach to obtaining the solutions. In the next section we begin defining an expression $\frac{\mathrm{d} a}{\mathrm{~d} X}=\mathscr{H}(a, X)$ which, together with Eq. (13) and the model proposed for the EoS, allows us to determine the phenomenological behavior of $F$ as a function of $X$.

\section{New parametrizations and kinetic k-essence Lagrangians}

As we have mentioned, the main goal of this research is to obtain a parametric expression relating $F$ and $X$ by means of the parametrization of the scale factor $a$, i.e., assuming that we know $F(a)$ and $X(a)$. In what follows, we display some proposed parameterizations [12-16] for the EoS of dark energy and their main drawbacks. The explicit expressions are

$$
\frac{\omega-\omega_{0}}{\omega_{a}}=\left\{\begin{array}{l}
z=\frac{1-a}{a}, \\
\frac{z}{1+z}=1-a, \\
\ln (1+z)=-\ln a, \\
\ln \left(1+\frac{z}{1+z}\right)=\ln (2-a),
\end{array}\right.
$$

where $\omega_{0}$ is the present value of the parameter $\omega, \omega_{a}$ should be adjusted to the observational data. In each parametrization $\omega_{0}$ represents the value of $\omega$ at the present time and if $\omega_{0}=$ -1 and $\omega_{a}=0$ they lead to the classical model $\Lambda \mathrm{CDM}$. The first parametrization represents a good fit for small $z$, but it has a serious problem explaining the observations for large $z(z>1)$. The second shows good behavior when $z<1$ and $z>1$. This is because, for $z<1, \omega$ has an approximately linear behavior while for $z \gg 1, \omega$ is bounded. In the parameterization proposed in [16], when $z \rightarrow \infty, w(z)$ becomes infinite, so this parameterization can only describe the behavior of dark energy when $z$ is not very large. In order to avoid this problem a modification was introduced in [17] assuming the form $w(z)=w_{0}+w_{a} \ln (1+z / 1+z)$. For future evolution, when $z \rightarrow 1,|w(z)|$ will grow rapidly and diverge, which is a nonphysical behavior of this EoS.

We show below explicitly the expression for $F(a)$ and $X(a)$ for the dark energy models proposed in [12-16], using Eq. (11) and Eq. (12); however, the reconstruction of the corresponding kinetic k-essence model, $F(X)$, will be done only for the new parameterization proposed by Feng and $\mathrm{Lu}$ in [16], which represents an improvement in the behavior of the dark energy EoS for a wide range of the redshift.

Using the parametrizations proposed in [12-16] in Eq. (11), we can solve for $X(a)$, finding

$$
\frac{X(a)}{X_{0}}=\left\{\begin{array}{l}
\left(\frac{\omega_{0}}{a}+1+\omega_{0}-\omega_{a}\right)^{2} a^{6\left(\omega_{a}-\omega_{0}\right)} e^{\left(\frac{6 \omega_{a}}{a}\right)}, \\
\left(1+\omega_{0}+\omega_{a}-\omega_{a} a\right)^{2} a^{-6\left(\omega_{0}+\omega_{a}\right)} e^{\left(6 \omega_{a} a\right)}, \\
\left(1+\omega_{0}-\omega_{a} \ln a\right)^{2} a^{-6 \omega_{0}} e^{\left(3 \omega_{a} \ln ^{2} a\right)}
\end{array}\right.
$$

Then using Eq. (12), the corresponding Lagrangian kinetic k-essence densities as a function of the scale factor are obtained:

$$
\frac{F(a)}{F_{0}}=\left\{\begin{array}{l}
\frac{\left(\omega_{a}+\left(\omega_{0}-\omega_{a}\right) a\right)^{-\frac{3}{\omega_{a}}} \exp \left(\frac{3 \omega_{a}}{a}\right)}{a^{3\left(\omega_{0}+1-\omega_{a}-\frac{1}{\omega_{a}}\right)}}, \\
\frac{\left(\omega_{0}+\omega_{a}-\omega_{a} a\right) \exp \left(3 \omega_{a} a\right)}{a^{3\left(1+\omega_{0}+\omega_{a}\right)}} \\
\frac{\left(\omega_{0}-\omega_{a} \ln a\right) \exp \left(\frac{3}{2} \omega_{a} \ln ^{2} a\right)}{a^{3\left(1+\omega_{0}\right)}} .
\end{array}\right.
$$


Using these equations we can reconstruct $F=F(X)$ by inverting the expression for $X(a)$, but we need $\frac{\mathrm{d} X}{\mathrm{~d} a} \neq 0$ to guarantee the existence of the inverse. In what follows, we will do an explicit reconstruction of $X$ for the parameterizations proposed in [17] and given by Eq. (14d). This ansatz has great interest in their behavior as a dark energy model, because it combines the advantages of the previous parameterizations. Also, this model has a future singularity at $z=-\frac{1}{2}$, which is a value bounded by the big bang. Depending on the dynamics, it may be adjusted to show a phantom-type behavior for a given redshift; however, in [21] it is discussed that the k-essence models entail problems in the case where the values of the adiabatic sound speed cross this barrier. This detail is dependent on the chosen parametrization and the action taken initially.

Imposing Eq. (12), we cannot explicitly obtain $F(X)$ for this particular model. However, if we manipulate this expression and solve an equation of the form $\frac{\mathrm{d} a}{\mathrm{~d} X}=\mathscr{H}(a, X)$ numerically, and we use Eq. (13), we can then obtain approximate values for $F(X)$ with $X>0$. We rewrite Eq. (11) as follows:

$\frac{\mathrm{d} a}{\mathrm{~d} X}=\mathscr{H}(a, X)=-\frac{a}{6 C_{\mathrm{s}}^{2}(a) X}$,

where the adiabatic sound speed squared $C_{\mathrm{s}}^{2}$ for the model given by Eq. (14d) is given by

$C_{\mathrm{s}}^{2}=\omega_{0}+\omega_{a} \ln (2-a)+\frac{\omega_{a} a}{3(2-a)\left(1+\omega_{0}+\omega_{a} \ln (2-a)\right)}$.

Then, introducing Eq. (18) into Eq. (17) we obtain

$$
\begin{aligned}
\frac{\mathrm{d} a}{\mathrm{~d} X} & =-\frac{a}{2 X} \\
& \times\left(\frac{\left(1+\omega_{0}+\omega_{a} \ln (2-a)\right)}{\left(\omega_{0}+\omega_{a} \ln (2-a)\right)\left(1+\omega_{0}+\omega_{a} \ln (2-a)\right)-\frac{\omega_{a} a}{a-2}}\right) .
\end{aligned}
$$

In [17] it was determined that the values which fit the observational data are $\omega_{0}=-1.0537$ and $\omega_{a}=0.2738$. Using these values, we plotted the behavior of $a=a(X)$ (see Fig. 1). This numerical solution was obtained using the Runge-Kutta method (see [18]) and it is the first step in the reconstruction process of the kinetic k-essence Lagrangian. In Fig. 2 the behavior of $F(X)$ as a function of $X$ is displayed, where the initial condition chosen was $a(X=1)=1$.

It is straightforward to see from Fig. 2 that the reconstructed k-essence Lagrangian is a decreasing function of the kinetic energy, $X$, of the field. Note that the reconstruction in $X=0$ is not possible due to the indetermination in the values of $F_{X}$, which can be appreciated in Eq. (6). From direct inspection of Eq. (5) we can conclude that

$$
\begin{aligned}
& \omega+1>0 \Rightarrow F_{X}<0, \\
& \omega+1<0 \Rightarrow F_{X}>0 .
\end{aligned}
$$

The above conditions imply that, if $F>0$, phantom behaviors can be obtained from kinetic k-essence Lagrangians which are increasing functions of $X$, whereas quintessence behaviors $(\omega>-1)$ are obtained from decreasing functions. On the other hand, from the conditions imposed on $C_{\mathrm{S}}^{2}$ [10], we obtain

$$
\begin{aligned}
& \omega>-1: F_{X X}>0, \\
& \omega<-1: F_{X X}<0 .
\end{aligned}
$$

So, the Lagrangian leading to an EoS in the range of quintessence will be decreasing functions of $X$ but with a positive second derivative, whereas for those which represent the phantom EoS, the Lagrangian is an increasing function of $X$, but with a negative second derivative or, in other words, it will be a concave function.

\section{Observational constraints}

In this section we use the parameterizations of Eqs. ((14a)(14d)) to obtain the best reconstruction of $F(X)$ based on observational data. In particular, we make use of the Pantheon data set [28] consisting of 1049 SNIa data points. The SNIa data enable us to constrain the luminosity distance $d_{L}(z)=$ $(1+z) r(z)$, where $r(z)$ is the comoving distance. We fit the SNIa with the cosmological model by minimizing the $\chi^{2}$ value defined by

$\chi_{\mathrm{SNIa}}^{2}=\Delta \boldsymbol{\mu}^{T} \cdot \mathbf{C}^{-1} \cdot \Delta \boldsymbol{\mu}$,

where $\Delta \boldsymbol{\mu}=\boldsymbol{\mu}-\boldsymbol{\mu}_{m}$ and $\boldsymbol{\mu}_{m}$ is a vector containing the distance modulus of the model for each redshift,

$\mu_{m}(z) \equiv 5 \log _{10}\left[d_{L}(z) / \mathrm{Mpc}\right]$

$\mathbf{C}$ is the uncertainty matrix defined by $\mathbf{C}=\mathbf{D}+\mathbf{C}_{\text {sys }}$, with D the statistical (diagonal) matrix and $\mathbf{C}_{\text {sys }}$ the systematic covariance matrix.

Actually, there is no need to use (11) and (12) to obtain $X(a)$ and $F(a)$ for a given $\omega(a)$. In fact, from (4) we know that

$F(a)=\omega(a) \rho(a)$,

and from (6) we know that

$4 \kappa X(a)=a^{6}(1+\omega(a))^{2} \rho(a)^{2}$. 
So, all we need is the expression for $\rho(a)$. From the mass conservation equation we can write

$\rho(z)=\rho_{0}(1+z)^{3} \exp \left(3 \int_{0}^{z} \frac{\omega(x)}{1+x} \mathrm{~d} x\right)$

where we have introduced the redshift $z$ defined through the relation $a=(1+z)^{-1}$.

\subsection{Model A}

Let us start with a first case, the one we called Model A, which is defined through its EoS parameter given by $w(z)=$ $w_{0}+w_{a} z$. Using Eq. (24) we obtain

$\rho(z)=\rho_{0}(1+z)^{3\left(1+w_{0}-w_{a}\right)} e^{3 w_{a} z}$,

and from Eq. (22) we get

$F(z)=\rho_{0}\left(w_{0}+w_{a} z\right)(1+z)^{3\left(1+w_{0}-w_{a}\right)} e^{3 w_{a} z}$.

Therefore, by using Eq. (23) we obtain

$4 \kappa X(z)=\rho_{0}^{2}\left(1+w_{0}+w_{a} z\right)^{2}(1+z)^{6\left(w_{0}-w_{a}\right)} e^{6 w_{a} z}$.

With these expressions we can plot $F(X)$ parametrically using the scale factor (or the redshift) as the parameter. The values for $w_{0}$ and $w_{a}$ in the plots are obtained from a test of the model against observational data, in this case a Type Ia supernova. The best fit to the SNIa data gives $\Omega_{m}=0.2 \pm 0.1, w_{0}=0.9 \pm 0.1$, and $w_{a}=0.8 \pm 0.2$, with $\chi_{\text {red }}^{2}=1.23$. In the first panel of Fig. 1 we display $F$ as a function of the redshift $z$, and in the other $X$ as a function of $z$. Combining these two functions, we plot in Fig. 2 the reconstructed $F(X)$ using the best fit values of the parameters.

A stability criterion demands that the sound speed be positive $c_{\mathrm{s}}^{2}>0$, which eventually translates into a restriction of the values of the EoS parameter $w(z)$. Using Eq. (7) we notice that $c_{\mathrm{s}}^{2}>0$ means $X^{\prime}<0$, or in terms of the derivative with respect to redshift, that $\mathrm{d} X / \mathrm{d} z>0$. From inspection in Fig. 1 for model $\mathrm{A}$, this never happens in the range $z \in[0.0,1.6]$. Notice that $F(X)$ is single valued and crosses from negative to positive values around $X \simeq 0.1$.

The same procedure is performed for the other three parameterizations:

Model B $\quad w(z)=w_{0}+w_{a} z /(1+z)$,

Model C $\quad w(z)=w_{0}+w_{a} \ln (1+z)$,

Model D $w(z)=w_{0}+w_{a} \ln (1+z /(1+z))$,

and the results are displayed in Table 1.
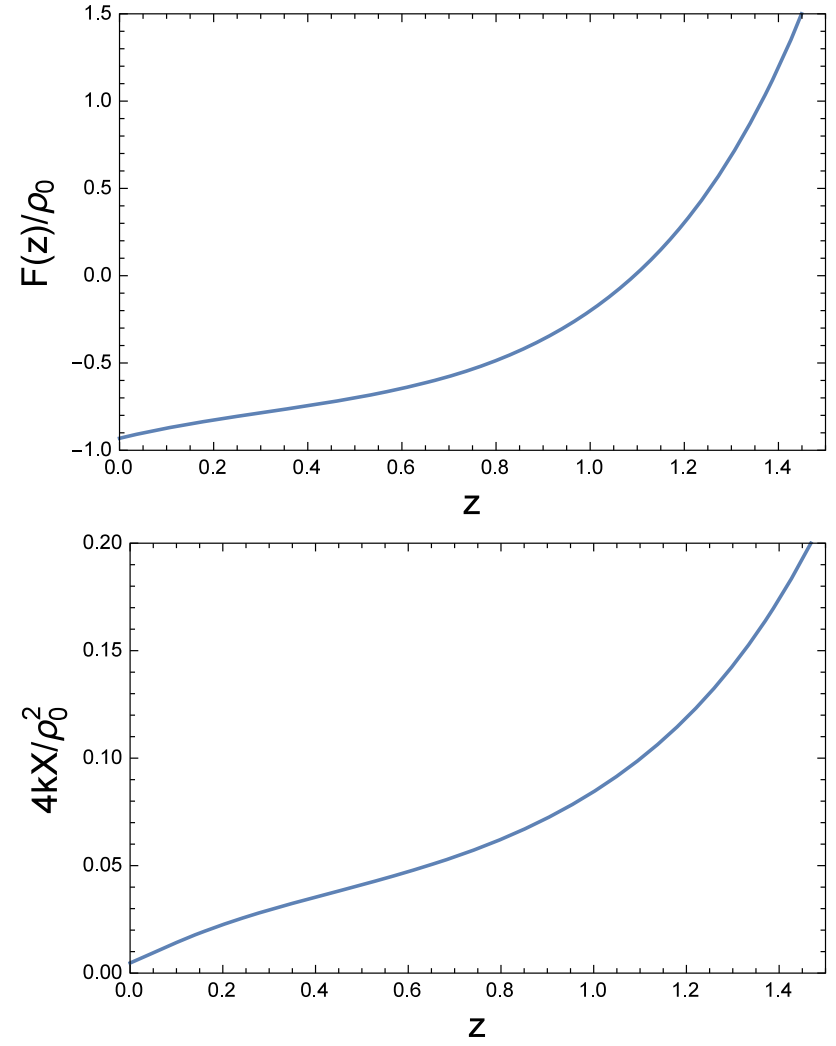

Fig. 1 Here we display $F$ and $X$ as a function of redshift based on Eqs. (26) and (27) for model A characterized by the parametrization $w_{0}+w_{a} z$

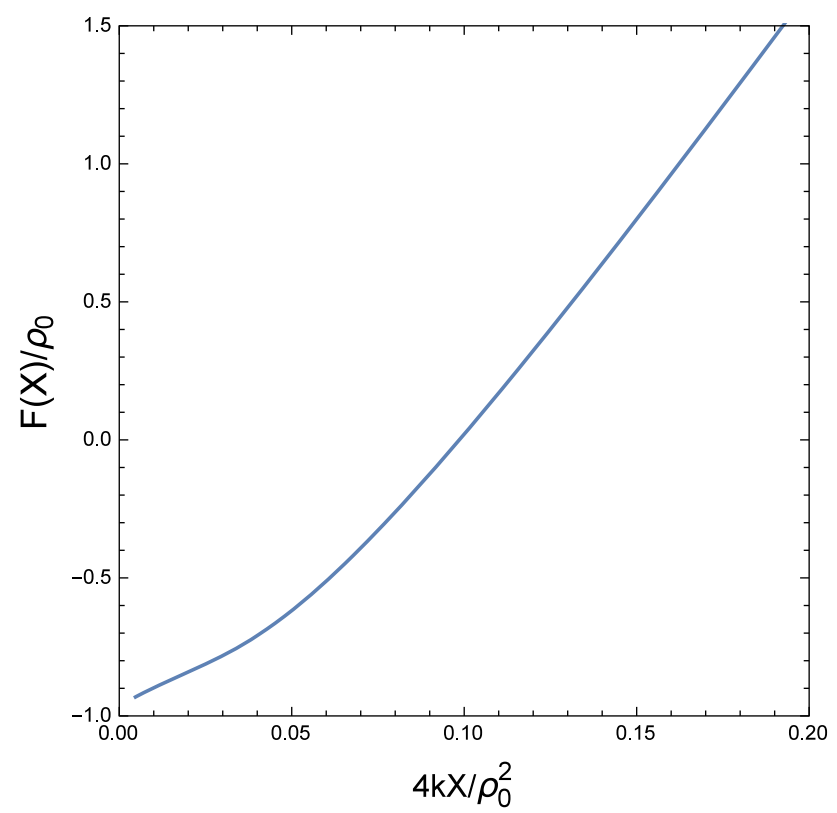

Fig. 2 Here the reconstructed $F(X)$ is shown based on the parametrization $w_{0}+w_{1} z$ with the best fit values of $w_{0}, w_{a}$ obtained from the SNIa data points 
Table 1 Summary of the result of the reconstruction for each model

\begin{tabular}{llll}
\hline & $\Omega_{m}$ & $\omega_{0}$ & $\omega_{a}$ \\
\hline Model A & $0.2 \pm 0.1$ & $-0.9 \pm 0.1$ & $0.8 \pm 0.2$ \\
Model B & $0.2 \pm 0.1$ & $-1.1 \pm 0.2$ & $1.3 \pm 0.6$ \\
Model C & $0.2 \pm 0.1$ & $-1.0 \pm 0.2$ & $1.0 \pm 0.4$ \\
Model D & $0.35 \pm 0.06$ & $-1.2 \pm 0.2$ & $-0.08 \pm 0.0$ \\
\hline
\end{tabular}

\subsection{Model B}

For model B we have the Chevallier-Polarski-Linder (CPL) parameterization $w(z)=w_{0}+w_{a} z /(1+z)$. From Eq. (24) we obtain

$\rho(z)=\rho_{0}(1+z)^{3\left(1+w_{0}+w_{a}\right)} \mathrm{e}^{-3 \frac{w_{a} z}{1+z}}$

and from Eq. (22) we get

$F(z)=\rho_{0}\left(w_{0}+\frac{w_{a} z}{1+z}\right)(1+z)^{3\left(1+w_{0}+w_{a}\right)} \mathrm{e}^{-3 \frac{w_{a} z}{1+z}}$.

Using Eq. (23) we obtain

$4 k X(z)=\rho_{0}^{2}\left(1+w_{0}+\frac{w_{a} z}{1+z}\right)^{2}(1+z)^{6\left(w_{0}+w_{a}\right)} \mathrm{e}^{-6 \frac{w_{a} z}{1+z}}$

Following an identical procedure to the one above, we first plot the expressions for $F(z)$ and $X(z)$ as functions of the redshift (Fig. 3), and then we plot the reconstructed $F(X)$ for the model. The values for $w_{0}$ and $w_{a}$ in the plots are obtained from a test of the model against observational data (see Table 1). Again, as in the previous case, the model is stable in the entire redshift range. Notice that, as in the previous model, the $F(X)$ function is single valued in the redshift range where the reconstruction is valid (Fig. 4)).

\subsection{Model C}

For model $\mathrm{C}$ we work with $w(z)=w_{0}+w_{a} \ln (1+z)$. From Eq. (24) we obtain

$\rho(z)=\rho_{0}(1+z)^{3\left(1+w_{0}\right)+\frac{3}{2} w_{a} \ln (1+z)}$,

and from Eq. (22) we get

$$
\begin{aligned}
F(z)= & \rho_{0}\left(w_{0}+w_{a} \ln (1+z)\right) \\
& (1+z)^{3\left(1+w_{0}\right)+\frac{3}{2} w_{a} \ln (1+z) .} .
\end{aligned}
$$

Using Eq. (23) we obtain

$$
\begin{aligned}
4 k X(z)= & \rho_{0}^{2}\left(1+w_{0}+w_{a} \ln (1+z)\right)^{2} \\
& (1+z)^{6 w_{0}+3 w_{a} \ln (1+z)} .
\end{aligned}
$$
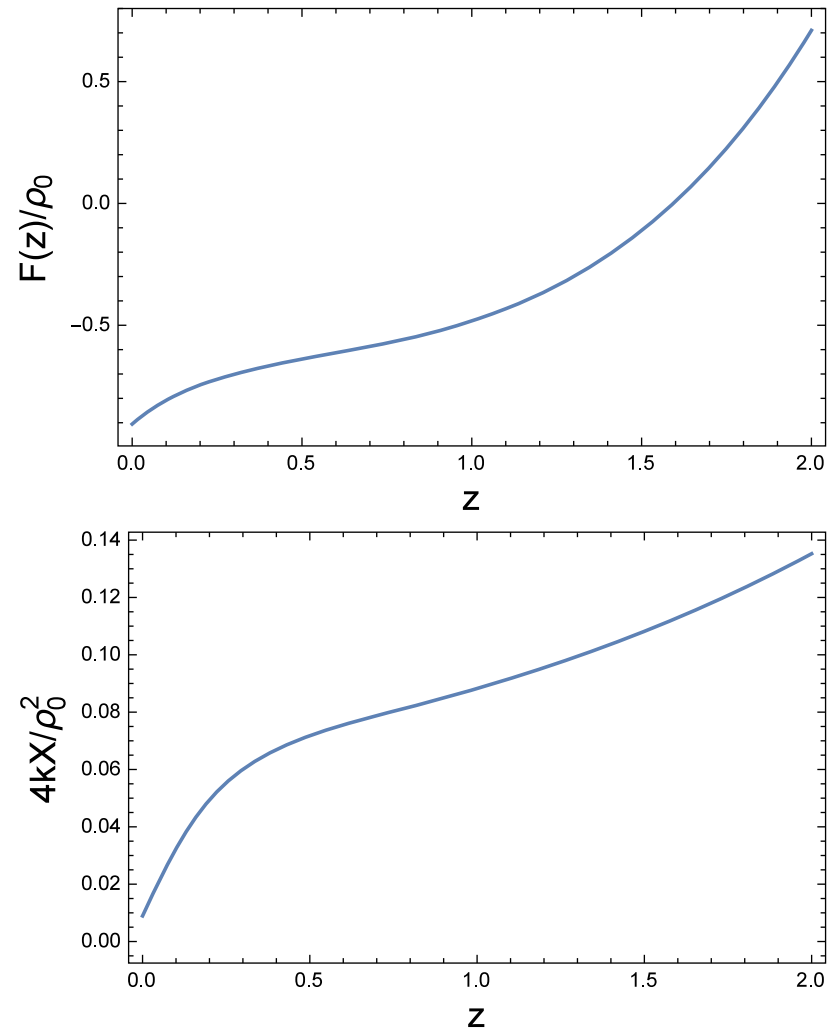

Fig. 3 Here we display $F$ and $X$ as a function of redshift based on Eqs. (30) and (31) for model B

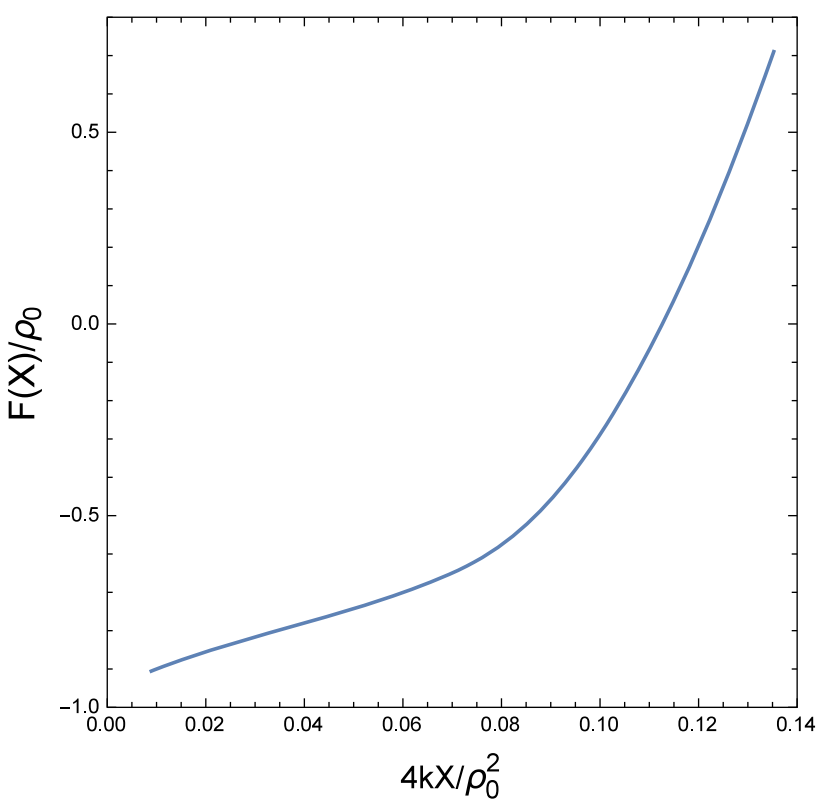

Fig. 4 Here the reconstructed $F(X)$ is shown based on the parametrization $w_{0}+\frac{w_{a} z}{1+z}$ with the best fit values of $w_{0}, w_{a}$ obtained from the SNIa data points

As before, we first plot the expressions for $F(z)$ and $X(z)$ as a function of redshift (Fig. 5), and then we plot the reconstructed $F(X)$ for the model. The best fit values for $w_{0}$ and $w_{a}$ are shown in Table 1. Again, in Fig. 6 we have a com- 

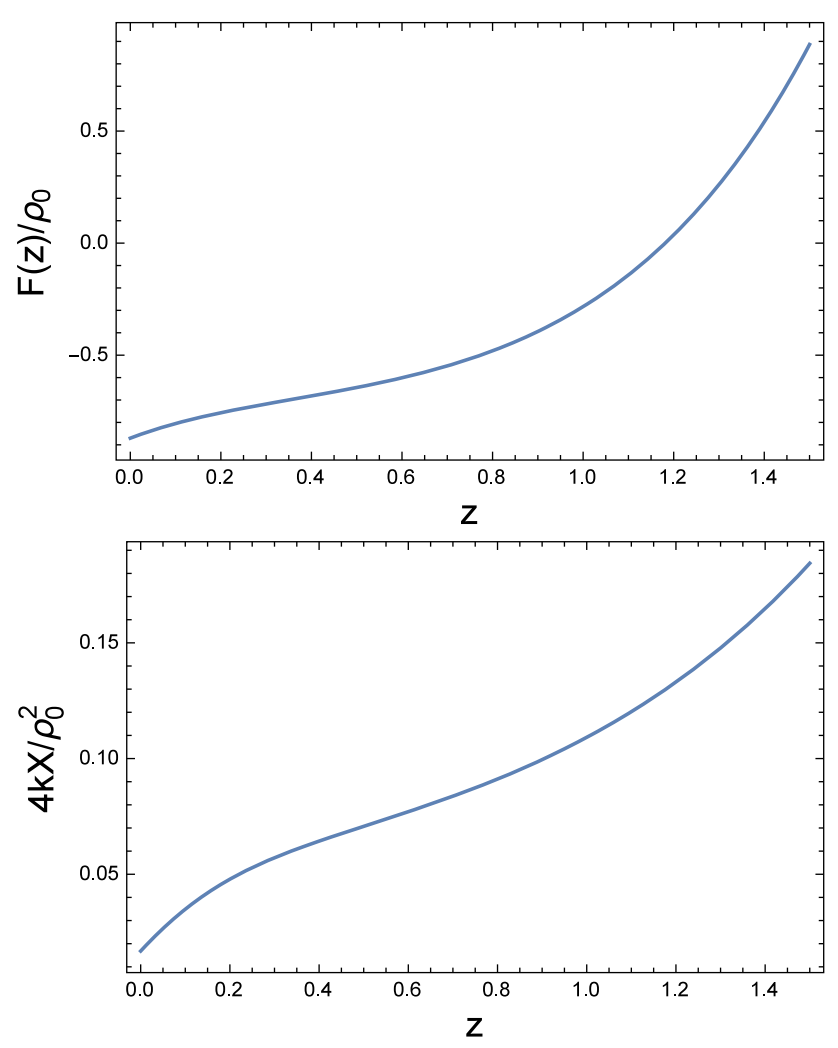

Fig. 5 Here we display $F$ and $X$ as a function of redshift based on Eqs. (33) and (34) for model C

pletely stable function $X(z)$. Notice that the stability region implies a single-valued $F(X)$ function, as is also the case in Model B and A.

\subsection{Model D}

For model D we work with $w(z)=w_{0}+\ln \left(1+\frac{z}{1+z}\right)$. From Eq. (24) we obtain

$\rho(z)=\rho_{0}(1+z)^{3\left(1+w_{0}\right)} \mathrm{e}^{g(z)}$,

where $g(z)$ is given by

$$
\begin{aligned}
g(z)= & \frac{\pi^{2} w_{a}}{4}-\frac{3 w_{a}[\ln (1+z)]^{2}}{2}+3 w_{a} \operatorname{Li}_{2}(-1-2 z) \\
& +3 w_{a} \ln (2(1+z)) \ln (1+2 z) .
\end{aligned}
$$

Here $\operatorname{Li}_{n}(z)$ is the ordinary polylogarithmic function [29]. From Eq. (22) we get $F(z)$ and using Eq. (23) we obtain $X(z)$. First we plot the expressions for $F(z)$ and $X(z)$ as functions of the redshift (Fig. 7), and then we plot the reconstructed $F(X)$ for the model. The best fit values of $w_{0}$ and $w_{a}$ for this model are shown in Table 1. Again, in Fig. 8 in the whole range of $z$ the speed of sound is positive. Although in the previous cases the function $X(z)$ was a increasing function, in this case $X(z)$ decreases with $z$. This implies that the

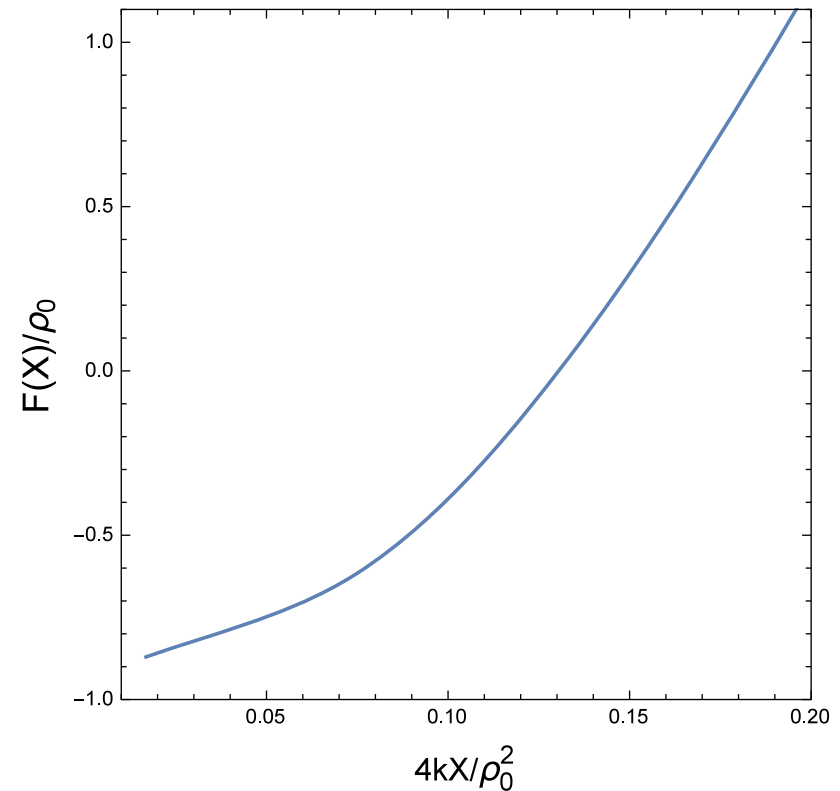

Fig. 6 Here the reconstructed $F(X)$ is shown based on the parametrization $w_{0}+\ln (1+z)$ with the best fit values of $w_{0}, w_{a}$ obtained from SNIa data points. The function is stable throughout the $z$ range. $\left(c_{\mathrm{s}}^{2}>0\right)$

reconstructed $F(X)$ decreases with $X$. Notice also that the stability region implies a single-valued $F(X)$ function as is also the case in all the previous cases.

\section{Conclusions and final remarks}

Many phenomenological parametrizations have been proposed to describe the behavior of the dark energy EoS as a function of the redshift. Our aim in this work has been to relate these phenomenological models with theoretical ones based on kinetic k-essence fields, which can be interpreted as a barotropic fluid [9] and are described by the rather simple Lagrangians found in previous investigations. We have shown the equations that can be solved numerically to reconstruct the Lagrangian density of the form $F=F(X)$ from an initial model $\omega=\omega(a)$. The first step of this reconstruction process is to connect the Lagrangian density $F$ and the kinetic energy $X$ with the scale factor. The interval of the definition of $X$ should be considered prior to the calculation of the approximation, always considering that $X>0$. Further, the equation $\frac{\mathrm{d} a}{\mathrm{~d} X}=\mathscr{H}(a, X)$ was solved to relate the kinetic energy $X$ and the Lagrangian $F$ by means of the scale factor. Next, we compared with the observational data using the Pantheon data set [28]. Thus, we obtained the best values of the parameters in agreement with the observations. Also, due to the impossibility of obtaining analytical solutions, we showed the graphical solution for each model described above. 

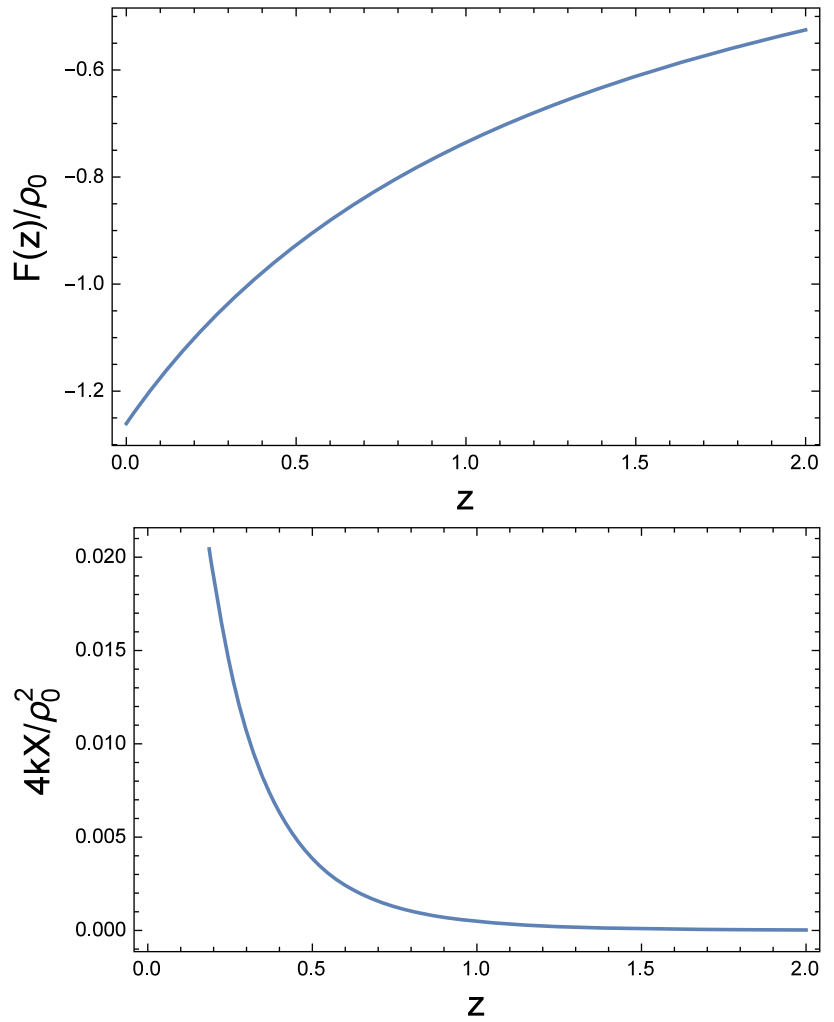

Fig. 7 Here we display $F$ and $X$ as functions of redshift derived in the text for model D

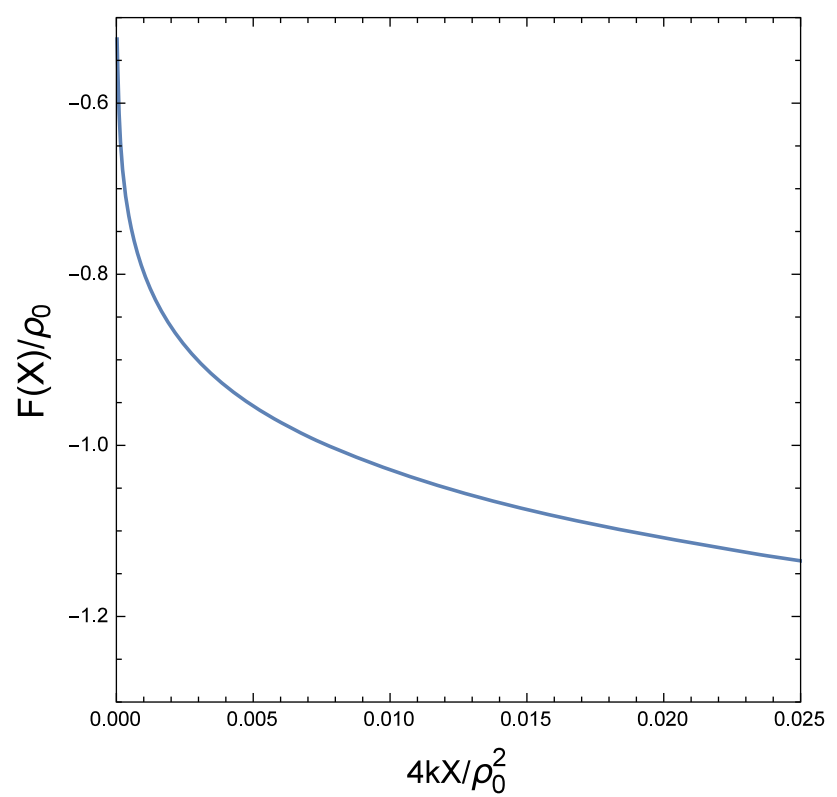

Fig. 8 Here the reconstructed $F(X)$ is shown based on the parametrization $w_{0}+w_{a} \ln (1+z /(1+z))$ with the best fit values of $w_{0}, w_{a}$ obtained from the SNIa data points. The black line indicates the segment in which $c_{\mathrm{s}}^{2}>0$

As a final summary we can conclude that the phenomenological equation of states proposed for the behavior of the dark energy may also appear as the effective behavior of a theoretical kinetic k-essence model. The results indicate that this equivalence can be justified in the whole redshift range supported by observational data.

Acknowledgements N. C. acknowledges the hospitality of the Instituto de Física y Astronomía of the Universidad de Valparaíso, where part of this work was done. This research was supported by CONICYT through FONDECYT Grant No. 1140238 (NC).

Open Access This article is distributed under the terms of the Creative Commons Attribution 4.0 International License (http://creativecomm ons.org/licenses/by/4.0/), which permits unrestricted use, distribution, and reproduction in any medium, provided you give appropriate credit to the original author(s) and the source, provide a link to the Creative Commons license, and indicate if changes were made.

Funded by $\mathrm{SCOAP}^{3}$.

\section{References}

1. C.G. Callan, J.M. Maldacena, Brane death and dynamics from the Born-Infeld action. Nucl. Phys. B 513, 198 (1998)

2. G.W. Gibbons, Born-Infeld particles and Dirichlet p-branes. Nucl. Phys. B 514, 603 (1998)

3. G.W. Gibbons, Aspects of Born-Infeld theory and string/M theory. Rev. Mex. Fis. 49S1, 19 (2003)

4. A. Sen, Tachyon matter. J. High Energy Phys. 0207, 065 (2002)

5. A. Sen, Rolling tachyon. J. High Energy Phys. 0204, 048 (2002)

6. C. Armendariz-Picon, T. Damour, V.F. Mukhanov, k-Inflation. Phys. Lett. B 458, 209-218 (1999)

7. R. Scherrer, Purely kinetic k-essence as unified dark matter. Phys. Lett. 93, 011301 (2004)

8. L.P. Chimento, Extended tachyon field, Chaplygin gas, and solvable k-essence cosmologies. Phys. Rev. D 69, 123517 (2004)

9. F. Arroja, M. Sasaki, A note on the equivalence of a barotropic perfect fluid with a k-essence scalar field. Phys. Rev. D 81, 107301 (2010)

10. R. de Putter, E.V. Linder, Kinetic k-essence and Quintessence Astropart. Phys. 28, 263 (2007)

11. R.J. Yang, J.Z. Qi, B.Z. Yang, Restrictions on purely kinetic kessence. Chin. Phys. Lett. 10, 109502 (2011)

12. A.R. Cooray, D. Huterer, Gravitational lensing as a probe of quintessence. Astrophys. J. 513, L95 (1999)

13. M. Chevallier, D. Polarski, Accelerating universe with scaling dark matter. Int. J. Mod. Phys. D 10, 213 (2001)

14. Q.J. Zhang, Y.L. Wu, Modelling time-varying dark energy with constraints from latest observations. Mod. Phys. Lett. A 27, $1250030(2012)$

15. E.V. Linder, Exploring the expansion history of the universe. Phys. Rev Lett. 90, 091301 (2003)

16. G. Efstathiou, Constraining the equation of state of the universe from distant Type Ia supernovae and cosmic microwave background anisotropies. Mon. Not. R. Astron. Soc. 310, 842-850 (2000)

17. L. Feng, T. Lu, A new equation of state for dark energy model. J. Cosmol. Astropart. Phys. 1111, 034 (2011)

18. J.R. Dormand, P.J. Prince, A family of embedded Runge-Kutta formulae. J. Comp. Appl. Math. 6, 19-26 (1980)

19. A. Sen, Reconstructing k-essence. J. Cosmol. Astropart. Phys. 0603, 010 (2006)

20. A. Rozas-Fernández, Kinetic k-essence ghost dark energy model. Phys. Lett. B 709, 313-321 (2012)

21. C. Bonvin, C. Caprini, R. Durrer, No go theorem for k-essence dark energy. Phys. Rev. Lett. 97, 081303 (2006) 
22. J. De-Santiago, J.L. Cervantes-Cota, Generalizing a unified model of dark matter, dark energy, and inflation with a noncanonical kinetic term. Phys. Rev. D 83, 063502 (2011)

23. J. Garriga, V.F. Mukhanov, Perturbations in k-inflations. Phys. Lett. B 458, 219 (1999)

24. D. Huterer, M.S. Turner, Prospect for probing the dark energy via supernova distance measurements. Phys. Rev. D 60, 081301 (1999)

25. J. Weller, A. Albrecht, Future supernovae observations as a probe of dark energy. Phys. Rev. D 65, 103512 (2002)

26. N. Bose, A.S. Majumbar, K-essence model of inflation, dark matter, and dark energy. Phys. Rev. D 79, 103517 (2009)
27. V.H. Cárdenas, N. Cruz, J.R. Villanueva, Testing a dissipative kinetic k-essence model. Eur. Phys. J. C 75, 148 (2015)

28. D.M. Scolnic et al., The complete light-curve sample of spectroscopically confirmed SNe Ia from Pan-STARRS1 and cosmological constraints from the combined pantheon sample. Astrophys. J. 859(2), 101 (2018)

29. M. Abramowitz, I.A. Stegun (eds.), Handbook of Mathematical Functions with Formulas, Graphs, and Mathematical Tables (Dover, New York, 1972) 